v.23, n.1, Especial do IV Simpósio de Nutrição de Animais de Companhia, p.11-12, 2018

\title{
EFEITO DO ARMAZENAMENTO EM CÂMARA CLIMÁTICA SOBRE A CONSERVAÇÃO DE DIETAS PARA CÃES COM DIFERENTES TAMANHOS DE EXTRUSADO E LOCAIS DE INCLUSÃO DE GORDURA
}

(Effect of storage in the climatic chamber on the conservation of diets for dogs with different sizes of extruded and fat inclusion sites) Emily M. Barros ${ }^{1}$, Daniele C. Lima ${ }^{1}$, Marley C. Santos ${ }^{1}$, Camilla M. M. Souza ${ }^{1}$, Nayara O. Carneiro ${ }^{1}$, Simone
G. Oliveira ${ }^{1}$

1'Departamento de Zootecnia - Universidade Federal do Paraná, Curitiba-PR.

E-mail: emilymatsunaga@ufpr.br

Resumo: Objetivou-se relacionar o tamanho de extrusado e a aplicação de gordura no condicionador ou recobrimento com a conservação de alimentos extrusados para cães, armazenados em câmara climática. Avaliaram-se dietas de mesma formulação, sendo: extrusado pequeno (PQ) com aplicação da metade da gordura no condicionador (CO) (PQCO); PQ com aplicação total da gordura no recobrimento (RE) (PQRE); extrusado grande (GR) com CO (GRCO) e GRRE. As dietas foram armazenadas em câmara climática com temperatura e umidade relativa do ar (URA) controladas por 20 semanas. Foram efetuadas análises de atividade de água ( $\mathrm{Aa}$ ), índice de peróxido (IP), umidade (UM), acidez, micotoxinas e colonização fúngica. Os dados foram submetidos a análise de regressão. As Aa e UM comparadas entre GRRE vs GRCO e PQRE vs PQCO apresentaram comportamento quadrático $(\mathrm{P}<0,001)$. A acidez apresentou aumento linear $(\mathrm{P}<0,001)$ para todos os tratamentos. Observou-se crescimento fúngico em todas as dietas. Alimentos para cães armazenados em condições controladas podem aumentar sua $\mathrm{Aa}$, umidade $\mathrm{e}$ acidez, afetando a conservação dos alimentos completos destinados a cães.

Palavras-chave: acidez; atividade da água; umidade

Abstract: The objective of this study was to relate the size of the kibble and the application of fat in the conditioner or coating with the preservation of extruded foods for dogs, stored in a climatic chamber. Diets of the same formulation were evaluated: small kibble (SM) with half fat application in the conditioner (CO) (SMCO); SM with total fat application in the coating (VE) (SMVE); large kibble (LA) with CO (LACO) and LAVE. Diets were stored in a climate chamber with temperature and relative humidity controlled for 20 weeks. Water activity (Aw), peroxide (PV), moisture, acidity, mycotoxins and fungal colonization were analyzed. Data were submitted to regression analysis. The Aw and moisture were compared between LAVE vs LACO and SMVE vs SMCO showed quadratic behavior $(\mathrm{P}<0.001)$. Acidity showed a linear increase $(P<0.001)$ for all treatments. Fungal growth was observed in all diets. Dog food stored under controlled conditions can increase your Aw, moisture and acidity, affecting the conservation of whole foods intended for dogs.

Keywords: acidity; moisture; water activity

Introdução: Variáveis do processo, como tamanho do extrusado e locais de aplicação de gordura podem impactar na conservação do alimento extrusado. Entretanto, a influência desses fatores ainda é pouco entendida. Portanto, o objetivo deste estudo foi relacionar o tamanho do extrusado e locais de aplicação (condicionador e recobrimento) de gordura com a conservação de dietas extrusadas para cães, estocadas em ambiente climatizado. 
Material e Métodos: Foram avaliadas quatro dietas de mesma formulação, sendo: PQ com aplicação da metade da gordura (4\%) no condicionador (CO) e a outra metade $(4 \%)$ por recobrimento (PQCO); PQ com aplicação total da gordura $(8 \%)$ no recobrimento (RE) (PQRE); GR com CO (GRCO) e GRRE. As dietas foram armazenadas em câmara climática com temperatura de $30^{\circ} \mathrm{C}$ e umidade relativa do ar (URA) de $75 \%$. Estas dietas foram divididas em potes plásticos com $120 \mathrm{~g}$ de ração/pote, fechados com plástico filme e contendo 60 furos/pote. Cada tratamento foi armazenado em 10 potes. Uma vez por semana foram realizadas análises de Aa, UM, IP e acidez. Foram realizadas ao início e ao final do experimento as seguintes análises: determinação de micotoxinas (Aflatoxinas B1, B2, G1 e G2 e Zearalenona por cromatografia líquida de alta eficiência) e crescimento fúngico. Ao final do experimento os resultados foram submetidos a análise de regressão.

Resultados e Discussão: A Aa e a UM das dietas apresentaram comportamento quadrático para todos os tratamentos $(P<0,05)$. Houve aumento linear $(P<0,001)$ na acidez de todos os tratamentos durante o período das 20 semanas. Isto é devido ao tempo em que as dietas permaneceram em altas temperaturas e URA. $O$ aumento da UM e Aa, de acordo com Krabbe (2009) está relacionado ao poder higroscópico dos extrusados e sua capacidade de absorver água do ambiente. Não houve valores positivos de IP em nenhuma amostra. Não houve evidência de micotoxinas nas dietas. Entretanto, houve o surgimento de fungos (1,14 UFC/g de Penicillium sp.) em todas as dietas. Fungos produzem micotoxinas quando estão sob situações de estresse, como alteração de temperatura e umidade (Haschek et al. 2002). É possível que a manutenção de dietas em condições controladas de temperatura e UM não tenham gerado estresse em fungos, o que resultaria na produção de micotoxinas.

Conclusão: Alimentos secos extrusados destinados a cães podem aumentar a $\mathrm{Aa}$, UM e acidez, quando submetidos a altas temperaturas e URA, independente do tamanho do extrusado e recobrimento de gordura, comprometendo a sua conservação.

Referências: HASCHEK, W. M.; VOSS, K. A.; BEASLEY, V. R. Selected mycotoxins affecting animal and human health. Handbook of Toxicologic Pathology, 2nd.; Academic Press: New York, 2002; pp 645-698.

KRABBE, E.L. Controle da atividade de água e produção de alimentos secos e semiúmidos. In: I Congresso Internacional e VIII Simpósio sobre nutrição de animais de estimação - CBNA. Campinas - SP, Anais...Maio de 2009. 